\title{
Presentación. \\ La relevancia social de la movilidad residencial
}

\author{
Ricardo Duque-Calvache \\ Universidad de Granada \\ ricardoduque@ugr.es
}

\section{Resumen}

Esta presentación tiene por objetivo contextualizar e introducir los trabajos incluidos en el número monográfico de la revista PAPERS sobre movilidad residencial, así como destacar la importancia de este fenómeno. La movilidad residencial es un elemento esencial de la naturaleza dinámica de nuestras ciudades, y sin tenerla en cuenta no es posible una adecuada comprensión de otras cuestiones socialmente relevantes, como las transformaciones de los barrios, el crecimiento urbano o la movilidad social. Las contribuciones a este número constituyen una panorámica de investigaciones empíricas sobre diferentes ámbitos que no solo dibujan el estado de la cuestión sino que también conectan el fenómeno con otras formas de cambio social.

Palabras clave: movilidad residencial; barrios; áreas metropolitanas

\section{Abstract. Presentation. Social Relevance of Residential Mobility}

This presentation aims to contextualize and introduce the articles included in the special issue of PAPERS on residential mobility, as well as to underline the importance of this phenomenon. Residential mobility is a key component of the dynamic nature of our cities, and if we do not take it into account, it would not be possible to achieve an adequate understanding of other relevant social problems, such as neighborhood changes, urban growth or social mobility. The papers in this issue include a number of empirical research studies with different scopes, and overall, they draw a picture of the state of the art and also connect the topic with other aspects of social change.

Keywords: residential mobility; neighborhoods; metropolitan areas 
Diversos investigadores han señalado la intensificación de la movilidad como una característica fundamental de las sociedades actuales. Los flujos de población son más numerosos que nunca, y también las distancias cubiertas se amplían a medida que los medios de transporte se vuelven más rápidos y accesibles. Aunque la movilidad no es un fenómeno nuevo, sus dimensiones actuales llevan a algunos a preguntarse si pueden suponer un cambio de paradigma, una forma de relación diferente entre la sociedad y el espacio que habitamos. Sin duda, fenómenos que implican desplazamientos masivos de población en el espacio revisten un gran interés, pero la atención dedicada a diferentes tipos de movimientos varía significativamente. En tanto que las migraciones internacionales son uno de los campos más profundamente estudiados en la actualidad, la investigación sobre movilidad residencial, compuesta por desplazamientos de menor recorrido, es significativamente inferior.

¿Por qué consideramos la movilidad residencial un fenómeno social de gran relevancia? En primer lugar, por sus efectos tangibles sobre el territorio, el espacio que habitamos. Los flujos de movilidad residencial han modelado la forma de nuestras ciudades produciendo y reproduciendo espacios y dinámicas cotidianas. En segundo lugar, por su conexión con la movilidad social: los cambios de vivienda implican en cierta manera una reubicación en la escala social (con frecuencia esta última es precisamente la razón del cambio), al tiempo que la estabilidad residencial puede ser considerada una limitación a las posibilidades de ascenso social (o, por el contrario, un asidero al que aferrarse). En tercer lugar, las decisiones acerca de la vivienda han sido explicadas desde planteamientos inicialmente vinculados a una concepción "naturalista» del ciclo vital, donde las familias se amoldan a los recursos disponibles. Estos modelos parten de factores principalmente económicos, por lo que se establece así una definición de situación donde el papel preponderante lo tienen los mercados y las dinámicas de oferta, demanda y precios. Sin embargo, las investigaciones internacionales en torno a la movilidad son crecientemente sensibles a variables con un carácter más sociológico, como la influencia de los barrios, las redes de apoyo o la familia, lo que refuerza nuestra convicción acerca de la necesidad de profundizar en el estudio de la movilidad residencial y en su interés para la sociología.

Este número especial de PAPERS reúne a algunos de los principales investigadores del fenómeno de la movilidad residencial en el ámbito estatal y a reputados expertos internacionales en la materia. Congregar a tales autores fue posible gracias a la celebración de un simposio sobre «Movilidad residencial en áreas metropolitanas», organizado por el proyecto «Procesos de reconfiguración social metropolitana» (CSO2014-55780-C3-3-P) del Plan Nacional de I + D. La calidad y variedad de las intervenciones presentadas en el encuentro impulsó la idea de lanzar un monográfico en torno a la cuestión, dado que hasta ahora no se había abordado en ninguna revista científica en el Estado español. El contenido de este número va a permitir a sus lectores conocer una panorámica general de la movilidad residencial, que incluye el origen y contenido del concepto, las fuentes disponibles para su estudio, el estado de la cuestión en 
nuestro entorno próximo, la conexión con otros procesos sociales y algunas de las líneas de trabajo en las que se seguirá trabajando en años venideros.

La primera cuestión relevante es la definición y cuantificación de la importancia del fenómeno, tarea que abordan en su texto Palomares-Linares, Feria y Susino. Es fundamental acotar con precisión la noción de movilidad residencial dentro de los diferentes flujos de movilidad, en especial para diferenciarla de las migraciones. La delimitación de las áreas metropolitanas, y su entendimiento en tanto que mercados de vivienda y trabajo, es el concepto que va a permitir articular tales diferencias. La definición y la medición están a su vez conectadas con la selección y tratamiento de las fuentes de datos para abordar su estudio.

Una vez establecidas estas bases conceptuales y metodológicas, es posible adentrarse en la comprensión del objeto de estudio y profundizar en las posibles explicaciones de su funcionamiento y evolución. William A.V. Clark aborda en su texto un repaso de los diferentes enfoques teóricos acerca de la movilidad residencial durante las últimas décadas, además de apuntar a nuevas líneas de interés para el presente y futuro de estos estudios. Si bien los investigadores difieren en sus planteamientos, todas las perspectivas comparten el intento de conectar las conductas de individuos y hogares con elementos estructurales, como los mercados de la vivienda o la configuración urbana. Duque, Torrado y Fuster hacen hincapié en la relevancia de los factores espaciales y contextuales al comprobar sus efectos sobre la movilidad de los individuos en el conjunto de las áreas metropolitanas españolas. Posteriormente, trabajando con datos desagregados por áreas, constatan la diversidad de situaciones y contextos locales, que apuntan a la necesidad de contemplar las dinámicas residenciales como flujos estrechamente vinculados a una realidad urbana compleja y heterogénea. Estos componentes estructurales permean y modulan las decisiones individuales.

El trabajo de Palomares-Linares y van Ham introduce la importancia de los barrios y el apego residencial, que estudian junto a indicadores del curso vital, la carrera residencial o la posición social, y evalúan su peso en las conductas de los hogares utilizando un modelo de regresión de conteo. Es destacable también su insistencia en diferenciar distintos grados de (in)movilidad, puesto que los detonantes y las explicaciones de las conductas hipermóviles no son necesariamente los mismos que nos van a permitir entender el sedentarismo. Tal vez cabe preguntarse si conviene pensar en términos de movilidad en general, o más bien de movilidades particulares. Sin ir más lejos, no podemos asumir que toda movilidad residencial es deseada, o que solamente es necesario contemplar la conducta residencial. El trabajo que presenta Módenes se focaliza en la inseguridad residencial, la percepción del riesgo de verse forzado a abandonar la vivienda, y realiza una comparativa con el entorno europeo. La transmisión familiar de seguridad residencial entre generaciones ha sido clave para frenar la inseguridad causada por la crisis económica y la precariedad laboral, pero se trata de una solución coyuntural, que no será replicable en futuras crisis. 
Esta conexión entre mercado laboral y residencial ha sido explorada también por Romaní, Casado y Lillo, que modelizan los factores explicativos de los desplazamientos residencia-trabajo y los cambios de domicilio dentro de los mercados locales de trabajo. Una de las conclusiones de su estudio es que la movilidad residencial tiende a alejar a los empleados de sus lugares de trabajo en lugar de acercarlos. Esta tendencia a la dispersión puede desarrollarse hasta derivar en fenómenos como el sprawl residencial o expansión urbana incontrolada, que García Coll y López Villanueva han caracterizado para el caso de la región metropolitana de Barcelona. El interés de estos movimientos centrífugos no estriba únicamente en la redistribución de población que suponen; también es crucial la transformación social y demográfica de los municipios ocasionada por la desigual participación en ellos de personas de diferentes edades e ingresos.

Estas dinámicas de movilidad que operan en la expansión urbana transforman también los municipios centrales, como da cuenta Andújar en su trabajo sobre el municipio de Madrid. Trabajando a escala inframunicipal, la autora identifica procesos de transformación y reproducción social en los diferentes barrios y el impacto de la crisis sobre ambos tipos de cambios. López-Gay coincide en la relevancia del periodo de crisis en la evolución de la movilidad en su trabajo sobre la región metropolitana de Barcelona, pero añade a esta coyuntura otro nivel contextual, conectado al desarrollo metropolitano y sus fases. Al estudiar las variaciones en la movilidad comprueba que, incluso cuando su intensidad se mantiene a nivel general, cambian los patrones territoriales del fenómeno. Uno de los factores impulsores de buena parte de los cambios es la inmigración procedente del extranjero, que es especialmente intensa en ciudades como Barcelona o Madrid. Sin embargo, estudiando el conjunto de la comunidad de Madrid, del Pino Artacho observa una convergencia entre los patrones de movilidad de la población procedente del extranjero una vez asentada con los del resto de habitantes de la región. Aunque señala también que no solo hay que contemplar las diferencias en las conductas residenciales de distintos grupos sociales, sino también la interacción entre grandes flujos, como ocurre con la centralización y la descentralización, cuyo desarrollo simultáneo puede producir una percepción errónea si no se observan separadamente.

El último artículo retoma una perspectiva internacional al afrontar el estudio de la movilidad residencial en Colombia. Villarraga y Módenes reflexionan acerca de los procesos de delimitación metropolitana a través de los flujos de movilidad cotidiana y el impacto de estos procedimientos en la medición de la movilidad residencial. En buena medida, este trabajo recupera los debates que se planteaban en el primer texto del monográfico al introducir la cuestión de la adaptación de estándares de investigación internacionales a un contexto muy diferente en su realidad social y urbana.

Retornando al título de esta presentación, esperamos que este número especial contribuya a divulgar la relevancia de la movilidad residencial y su conexión con otras problemáticas demográficas y urbanas, así como con la 
movilidad social. Al situarlo como un objeto de interés sociológico no pretendemos delimitar un campo acotado para la sociología, máxime si tenemos en cuenta que este número ha sido posible gracias a la participación de geógrafos, economistas y demógrafos. Pero sí queremos defender que las pautas de movilidad son sociales — una manifestación más del juego de acción y estructura- $\mathrm{y}$ que las distintas dinámicas residenciales modelan de manera muy relevante los entornos en que vivimos y las relaciones sociales en tales espacios. 